\title{
The IH-7-Gap Resonators of the Munich Accelerator for Fission Fragments (MAFF) LINAC
}

\author{
H.Bongers, S.Emhofer, D.Habs, T.Sieber, LMU München, D-85748 Garching, Germany \\ O.Kester, GSI, Darmstadt, Germany and CERN, Geneva, Switzerland \\ U.Ratzinger, A. Schempp, IAP Frankfurt, D-60325 Frankfurt/Main, Germany
}

\section{Abstract}

The LINAC of the new Munich Accelerator for Fission Fragments (MAFF), which is currently under construction at the new high flux reactor FRM II $[1,2]$, will deliver intense beams of neutron-rich fission fragments in order to produce new super-heavy elements with long halftimes and to study r-process nuclei [3]. The overall length of the LINAC is restricted to approximately $20 \mathrm{~m}$. To reach energies at the Coulomb Barrier with such a short LINAC very effective acceleration is mandatory. To obtain the proposed final energies in the range of 3.7 $\mathrm{MeV} / \mathrm{u}$ to $5.9 \mathrm{MeV} / \mathrm{u}$, the ions are charge bred to $\mathrm{A} / \mathrm{q}<$ 6.3 before acceleration and IH-structures with high shunt impedances and high effective accelerating fields are used for all LINAC components. The general concept of the LINAC and the IH-7-gap structures used to achieve the high flexibility in the end energies in particular will be presented.

\section{THE MAFF-LINAC}

The MAFF-LINAC [4] is planned to accelerate heavy neutron rich fission fragments produced in an $1.2 \mathrm{~g}{ }^{235} \mathrm{U}$ target at the FRM II. The available neutron flux of $1.5^{*} 10^{14} \mathrm{n} / \mathrm{cm}^{2} \mathrm{~s}$ of the reactor at the target results in a fission rate of $10^{14} / \mathrm{s}$. Intensities of several $10^{11}$ ions $/ \mathrm{s}$ for ${ }^{91} \mathrm{Kr},{ }^{132} \mathrm{Sn}$ or ${ }^{144} \mathrm{Cs}$ e.g. are expected after mass seperation. After separation, the ions are charge bred in an ECRIS and then injected into the LINAC. Due to the low energy spread from the ECRIS, only one magnetic deflector is necessary for the $\mathrm{A} / \mathrm{q}$ selection. The LINAC itself consists of several distinct structures:

The first section of the MAFF LINAC is proposed to be an IH-RFQ, in which two opposing girders are mounted and the cavity is exited in the $\mathrm{TE}_{1,1,1}$ mode at 101.28 MHz (Duty factor:10\%). The girders carry equidistant interdigital holders on which the quadrupole electrodes are fixed. A similar device at $37 \mathrm{MHz}$ operates as high current injector at GSI [5].

The booster section consists of three IH-structures to accelerate the beam from $300 \mathrm{keV} / \mathrm{u}$ to $4.15 \mathrm{MeV} / \mathrm{u}$ or $5.40 \mathrm{MeV} / \mathrm{u}$ respectively. Tank 1 , which contains two inner tank quadrupole triplets, is still operated at 101.28 $\mathrm{MHz}$, after this tank the frequency doubles to 202.56 MHz. Tank 3 can be switched on or off. In this way the beam can be injected into the energy variable structure of the LINAC with two different energies. The final energy variation is done by two identical IH-7-gap structures, which accelerate or decelerate the ion beam from the two different injection energies to the desired final energy. The entire range of $3.7 \mathrm{MeV} / \mathrm{u}$ to $5.9 \mathrm{MeV} / \mathrm{u}$ can be covered by these two coupled IH-7-gap resonators.

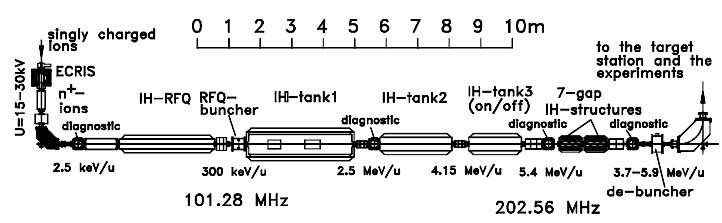

Figure 1: Overview of the MAFF LINAC.

\section{THE IH-7-GAP STRUCTURES}

\subsection{General}

The energy variable section of the LINAC consists of two identical IH-7-gap structures placed after the booster and operated at $202.56 \mathrm{MHz}$. To increase the efficiency of these 7-gap structures, compared to the split ring resonators used e.g. for REX-ISOLDE [6], the resonators are designed as IH-structures. Due to the higher shunt impedance of $\mathrm{IH}$-structures a higher resonator voltage in combination with a very compact design can be achieved with the same generator power compared to split ring resonators.

The booster injects the ions either at $4.15 \mathrm{MeV} / \mathrm{u}$ or $5.40 \mathrm{MeV} / \mathrm{u}$ into the IH-7-gap resonators by switching tank 3 off or on (see fig. 1). This beam is then accelerated or decelerated to the final energy. Therefore it is necessary that each of the two IH-7-gap structures is able to accelerate or decelerate over a range of at least \pm 0.31 $\mathrm{MeV} / \mathrm{u}$ with a sufficient transit time factor.

Furthermore in beam dynamics the combined $0^{\circ}$ synchronous particle structure (KONUS) is used for the center gaps. This means that the mean particle of the bunch will reach the center gap of each resonator at $0^{\circ}$ phase of the incoupled RF. This results in an overall higher transit time factor (TTF) over the whole energy range. 


\subsection{Design Layout}

Since the resonators are used both for efficient acceleration and deceleration respectively, they are designed identically with constant cell and gap lengths. Based on the fact that the energy would be between 3.7 $\mathrm{MeV} / \mathrm{u}$ and $5.9 \mathrm{MeV} / \mathrm{u}$ and $\mathrm{A} / \mathrm{q}$ is expected to be $<6.3 \mathrm{a}$ cell length of $74 \mathrm{~mm}$ at $202.56 \mathrm{MHz}$ was chosen, which corresponds to a design speed of $\beta=0.1$.

This results in an total length of $518 \mathrm{~mm}$ for the seven cells per resonator. The inner tank length is $520 \mathrm{~mm}$ and the overall outside length is $646 \mathrm{~mm}$ for each resonator. A drift tube length of $50 \mathrm{~mm}$ and an aperture of $20 \mathrm{~mm}$ were used. This still provides transit time factors in the range of 0.88 to 0.92 and even in the case of deceleration from 4.15 MeV/u to $3.7 \mathrm{MeV} / \mathrm{u}$ (biggest mismatch between design speed and actual bunch speed) a sufficient energy transfer can be provided at the resonator ends. To obtain the desired operation frequency of $202.56 \mathrm{MHz}$ the tank was planned to be $310 \mathrm{~mm}$ wide and $410 \mathrm{~mm}$ high and will be tuned by capacitive plungers during operation.

In order to determine the values for the cavity characteristics necessary to build a model of one resonator, extensive MAFIA calculations have been performed. These simulations of the model showed that a quality factor of 15000 to 18000 and a shunt impedance of approximately $180 \mathrm{M} \Omega / \mathrm{m}$ can be expected for the power resonators.

These calculations also show that $2.8 \mathrm{MV}$ total resonator voltage per resonator can be expected for the proposed IH-7-Gap resonators at $150 \mathrm{M} \Omega / \mathrm{m}$ shunt impedance, $100 \mathrm{~kW}$ generator power and $200 \mathrm{MHz}$ resonator frequency.

According to LINAC and LORASR calculations of the beam dynamics, only $2.2 \mathrm{MV}$ per resonator are necessary to cover the entire range of final energies. Assuming 150 $\mathrm{M} \Omega / \mathrm{m}$ shunt impedance, this voltage can be reached with a comparably low rf-power of $63 \mathrm{~kW}$. Higher power would not result in significant more efficient acceleration due to too extreme phase shifts (see fig. 2).

\subsection{Beam Dynamics}

Depending on the differences between injection energy, desired exit energy and design speed of the ions, the phase of the bunch will vary significantly from gap to gap (s. fig. 2). Since the mean particle of the bunch will reach the center of each resonator at $0^{\circ}$ phase, the phase switches its sign at the center gap. Therefore the beam is always longitudinally defocused in either the first three gaps of each resonator or in the last three gaps depending on whether the ions are accelerated or decelerated. However, this defocusing effect is mainly compensated by the fact that the beam will be focused in the corresponding three gaps. The energy spread and emmitance growth is low (see fig. 4).

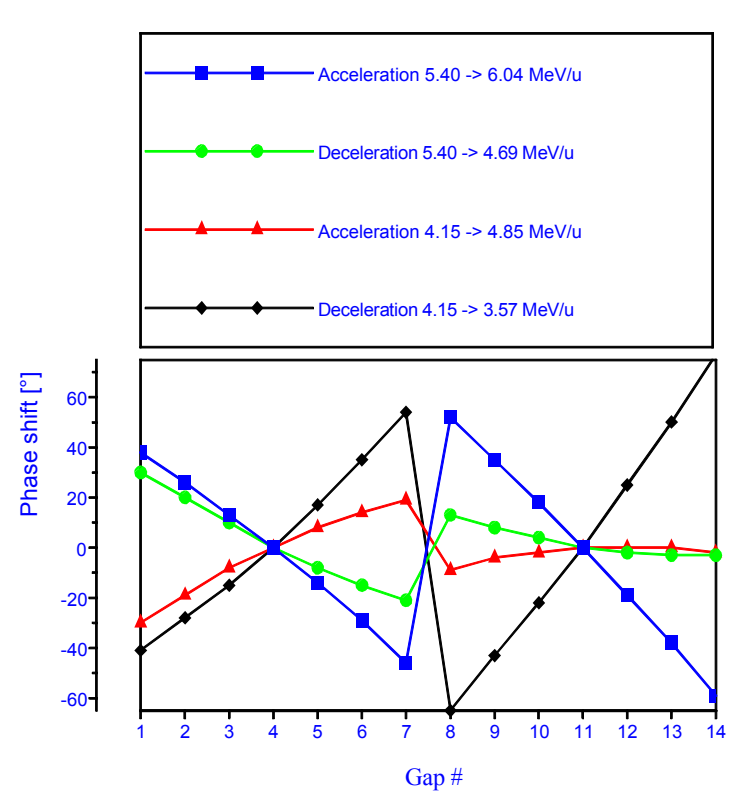

Figure 2: Phase shift of the ion bunch in relation to the synchronous particle.

This phase shift has a significant influence on the energy that can be transferred to the particles in each gap. In the case of deceleration to $3.7 \mathrm{MeV} / \mathrm{u}$ e.g. the TTFs of the center gap and last gap of the second resonator differ only by $0.4 \%$, but the value of $\mathrm{dW}$ decreases from 51.9 $\mathrm{keV} / \mathrm{u}$ to $8.0 \mathrm{keV} / \mathrm{u}$ (see fig. 3).

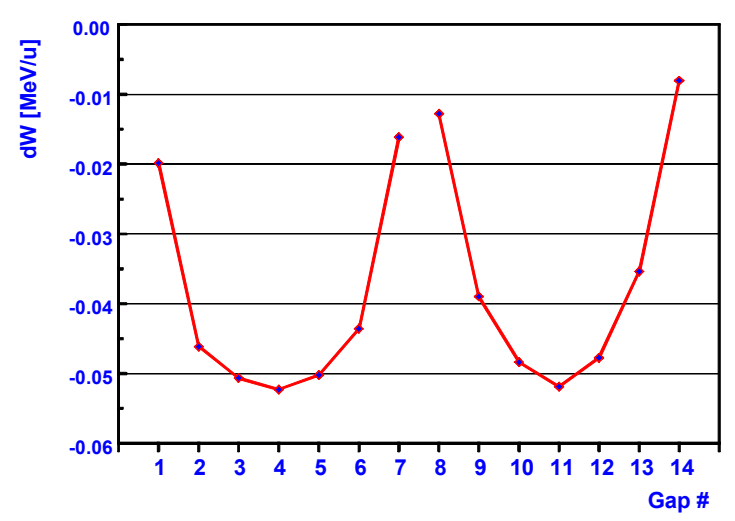

Figure 3: Effective energy transfer in the individual gaps for deceleration from 4.15 MeV/u to $3.70 \mathrm{MeV} / \mathrm{u}$ )

Using the LORASR code (U. Ratzinger) to calculate the beam dynamics, leads to even better results (more efficient acceleration, deceleration in particular). While the LINAC code simplifies the gap voltage distribution according to the distribution of split ring resonators to a ratio of $1: 2: 2: 2: 2: 2: 1$ LORASR code can use individual gap voltage values for each gap, which were derived from simulations and verified by measurements of the model. Also the different TTFs, depending on the distance of the tracked particle to the axis, are taken into account. 


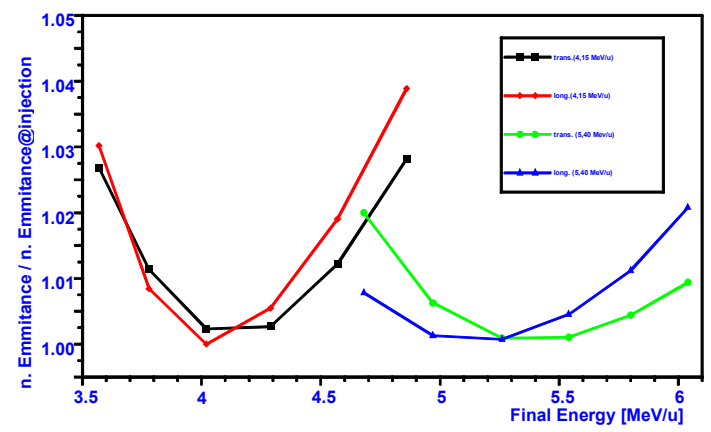

Figure 4: Emmitance growth in dependence of the final energies.

One advantage of IH-structures is that they do not have a flat gap voltage distribution (depending on the undercut design), but the gap voltage increases towards the center gap (s. fig. 6). Therefore the phase is $0^{\circ}$ in the gap with the highest effective field and the defocusing effects at the first and last gaps are reduced. Furthermore the resonator designs with higher voltage peaks have higher shunt impedances too.

\subsection{Measurements}

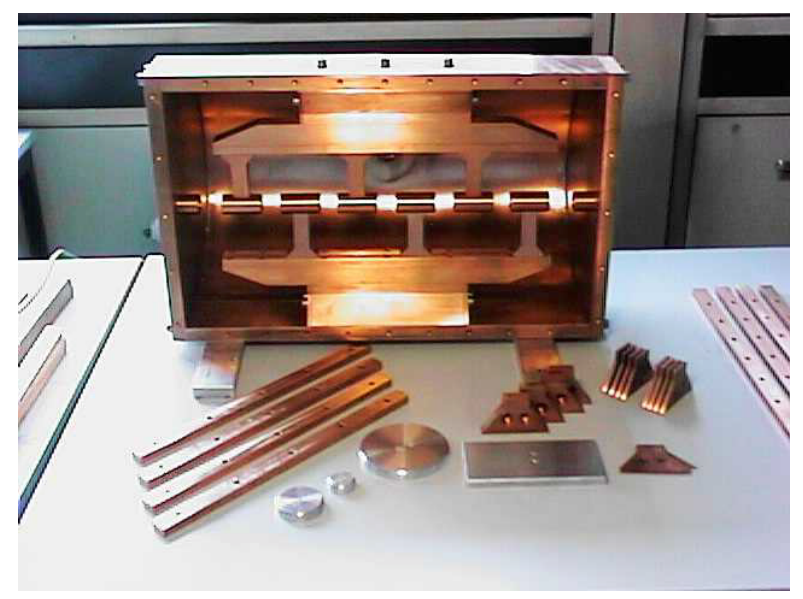

Figure 5: Model with different plungers, copper blocks and bars to change the undercuts and vary the flanges of the half-shells.

A 1:1 model has been build and used to optimize the design parameters and verify the MAFIA calculations. The model was built in such a way that the undercut lengths, height of the tank and the tuning plungers could easily be modified (s. fig. 5). In respect to resonance frequencies, resonance modes, dependency of the resonator characteristics on variation of the geometry, the measurement and voltage distributions are in good agreement with the calculations.

Due to the fact that the flexible design of the model results in various damping effects the proposed quality factor and the shunt impedance could not be verified yet. But by experiences with similar models and $\mathrm{IH}$-structures allready built it is reasonable to expect values of $\mathrm{Q}=18000$ and shunt impedances of $180 \mathrm{M} \Omega / \mathrm{m}$.

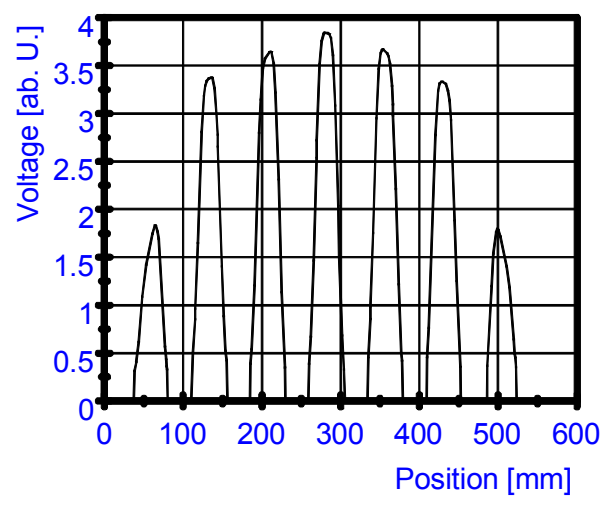

Figure 6: Measurement of the voltage distribution at the axis. The higher gap voltage in the center gap is clearly visible.

\subsection{Current Status}

Design and beam dynamic simulations with MAFIA, LINAC and LORASR have been performed and verified by measurements of the model. A 1:1 model of one of the resonator was built and successfully tested. One prototyp of a power-resonator is under construction. A test beam line for energy tests is currently prepared at the tandem lab in Munich.

\section{REFERENCES}

[1] O. Kester, D. Habs et al., Nucl. Instr. and Meth. B 139 (1998) 28

[2] Munich Accelerator for Fission Fragments (MAFF), Physics case and technical description, ed. by D.Habs et al., October 1998

[3] P. Thirolf, D. Habs et al., Nucl. Instr. and Meth. B 126 (1997) 242

[4] O. Kester et al., Proc. of the LINAC 98, Chicago, p 112

[5] U. Ratzinger et al., Nucl. Instr. and Meth., A328 (1993) 270

[6] R. von Hahn et al., proc of the EPAC2000, Wien, 2000 